\title{
The FIRST experiment at GSI
}

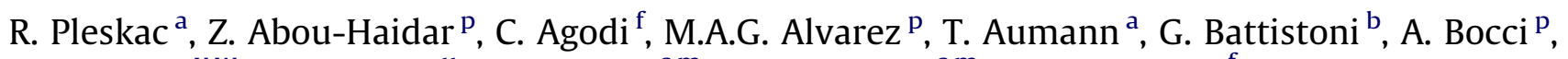
T.T. Böhlen ${ }^{\mathrm{v}, \mathrm{w}}$, A. Boudard ${ }^{\mathrm{u}}$, A. Brunetti ${ }^{\mathrm{c}, \mathrm{m}}$, M. Carpinelli ${ }^{\mathrm{c}, \mathrm{m}}$, G.A.P. Cirrone ${ }^{\mathrm{f}}$, M.A. Cortes-Giraldo ${ }^{\mathrm{q}}$, G. Cuttone ${ }^{f}$, M. De Napoli ${ }^{d}$, M. Durante ${ }^{\mathrm{a}}$, J.P. Fernández-García ${ }^{\mathrm{q}}$, C. Finck $^{\mathrm{r}}$, B. Golosio ${ }^{\mathrm{c}, \mathrm{m}}$, M.I. Gallardo ${ }^{\mathrm{q}}$, E. Iarocci ${ }^{e, j}$, F. Iazzi ${ }^{\text {h,k }}$, G. Ickert ${ }^{a}$, R. Introzzi ${ }^{\text {h }}$, D. Juliani ${ }^{r}$, J. Krimmer ${ }^{t}$, N. Kurz $^{\text {a }}$, M. Labalme ${ }^{\text {s }}$, Y. Leifels ${ }^{a}$, A. Le Fevre ${ }^{a}$, S. Leray ${ }^{\text {u }}$, F. Marchetto ${ }^{\text {h }}$, V. Monaco ${ }^{\text {h,l }}$, M.C. Morone ${ }^{\mathrm{i}, \mathrm{n}}$, P. Oliva ${ }^{\mathrm{c}, \mathrm{m}}$, A. Paoloni ${ }^{\mathrm{e}}$, L. Piersanti e,j, J.M. Quesada q, G. Raciti ${ }^{\mathrm{f}}$, N. Randazzo ${ }^{\text {f }}$, F. Romano ${ }^{\mathrm{f}, \mathrm{o}}$, D. Rossi ${ }^{\mathrm{a}}$, M. Rousseau ${ }^{\mathrm{r}}$, R. Sacchi ${ }^{\text {h,l }}$, P. Sala ${ }^{b}$, A. Sarti ${ }^{e, j}$, C. Scheidenberger ${ }^{a}$, C. Schuy ${ }^{a}$, A. Sciubba ${ }^{e, j}$, C. Sfienti ${ }^{x}$, H. Simon $^{\text {a }}$, V. Sipala ${ }^{\mathrm{d}, \mathrm{m}}$, E. Spiriti ${ }^{\mathrm{g}}$, L. Stuttge ${ }^{\mathrm{r}}$, S. Tropea ${ }^{\mathrm{f}}$, H. Younis ${ }^{\mathrm{h}, \mathrm{k}}$, V. Patera ${ }^{\mathrm{e}, \mathrm{j}, *}$

${ }^{a}$ GSI Helmholtzzentrum für Schwerionenforschung, Darmstadt, Germany

${ }^{\mathrm{b}}$ Istituto Nazionale di Fisica Nucleare - Sezione di Milano, Italy

' Istituto Nazionale di Fisica Nucleare - Sezione di Cagliari, Italy

${ }^{\mathrm{d}}$ Istituto Nazionale di Fisica Nucleare - Sezione di Catania, Italy

e Istituto Nazionale di Fisica Nucleare - Laboratori Nazionali di Frascati, Via E. Fermi 40, I-00044 Frascati, Italy

${ }^{\mathrm{f}}$ Istituto Nazionale di Fisica Nucleare - Laboratori Nazionali del Sud, Italy

${ }^{\mathrm{g}}$ Istituto Nazionale di Fisica Nucleare - Sezione di Roma 3, Italy

${ }^{\mathrm{h}}$ Istituto Nazionale di Fisica Nucleare - Sezione di Torino, Italy

${ }^{\mathrm{i}}$ Istituto Nazionale di Fisica Nucleare - Sezione di Roma Tor Vergata, Italy

j Dipartimento di Scienze di Base e Applicate per l'Ingegneria, "La Sapienza" Università di Roma, Italy

${ }^{\mathrm{k}}$ Dipartimento di Fisica, Politecnico di Torino, Italy

${ }^{1}$ Dipartimento di Fisica, Universita' di Torino, Italy

' Universita' di Sassari, Italy

${ }^{\mathrm{n}}$ Dipartimento di Biopatologia e Diagnostica per Immagini, Universita' di Roma Tor Vergata, Italy

"Centro Studi e Ricerche e Museo Storico della Fisica "Enrico Fermi", Roma, Italy

P CNA, Sevilla, Spain

${ }^{\mathrm{q}}$ Departamento de Fisica Atomica, Molecular y Nuclear, University of Sevilla, 41080-Sevilla, Spain

${ }^{\mathrm{r}}$ Institut Pluridisciplinaire Hubert Curien, Strasbourg, France

${ }^{s}$ LPC-Caen, ENSICAEN, Universit de Caen, CNRS/IN2P3, Caen, France

${ }^{\mathrm{t}}$ IPN-Lyon, Universit de Lyon, Universit Lyon 1, CNRS/IN2P3, Villeurbanne, France

${ }^{u}$ CEA-Saclay, IRFU/SPhN, Gif sur Yvette Cedex, France

${ }^{v}$ European Organization for Nuclear Research CERN, Geneva, Switzerland

${ }^{\mathrm{w}}$ Medical Radiation Physics, Karolinska Institutet and Stockholm University, Stockholm, Sweden

x Universitat Mainz Johann-Joachim-Becher, Mainz, Germany

\section{A R T I C L E I N F O}

\section{Article history:}

Received 28 October 2011

Received in revised form

10 February 2012

Accepted 11 February 2012

Available online 27 February 2012

\section{Keywords:}

Hadrontherapy

Fragmentation

Nuclear physics

Elementary-particle

\begin{abstract}
A B S T R A C T
The FIRST (Fragmentation of Ions Relevant for Space and Therapy) experiment at the SIS accelerator of GSI laboratory in Darmstadt has been designed for the measurement of ion fragmentation crosssections at different angles and energies between 100 and $1000 \mathrm{MeV} /$ nucleon. Nuclear fragmentation processes are relevant in several fields of basic research and applied physics and are of particular interest for tumor therapy and for space radiation protection applications.

The start of the scientific program of the FIRST experiment was on summer 2011 and was focused on the measurement of $400 \mathrm{MeV} /$ nucleon ${ }^{12} \mathrm{C}$ beam fragmentation on thin $(8 \mathrm{~mm})$ graphite target.

The detector is partly based on an already existing setup made of a dipole magnet (ALADiN), a time projection chamber (TP-MUSIC IV), a neutron detector (LAND) and a time of flight scintillator system (TOFWALL). This pre-existing setup has been integrated with newly designed detectors in the Interaction Region, around the carbon target placed in a sample changer. The new detectors are a
\end{abstract}

\footnotetext{
* Corresponding author at: Istituto Nazionale di Fisica Nucleare - Laboratori Nazionali di Frascati, Via E. Fermi 40, I-00044 Frascati, Italy. Tel.: + 39 0694032795; fax: + 390694032427.

E-mail address: vincenzo.patera@lnf.infn.it (V. Patera).
} 
Experimental methods Instrumentation scintillator Start Counter, a Beam Monitor drift chamber, a silicon Vertex Detector and a Proton Tagger scintillator system optimized for the detection of light fragments emitted at large angles.

In this paper we review the experimental setup, then we present the simulation software, the data acquisition system and finally the trigger strategy of the experiment.

(c) 2012 Elsevier B.V. All rights reserved.

\section{Introduction}

Particle therapy is a rapidly expanding field in cancer therapy, and generally exploits protons or carbon ions. Carbon ions combine significant advantages both in the physical dose-depth deposition pattern and in the biological effectiveness and may represent a significant breakthrough in radiotherapy [1]. Nuclear fragmentation cross-sections, as well as algorithms that deal with the transport of charged particle in matter, are essential for accurate treatment planning, as only roughly $50 \%$ of the heavy ions directed to the patient actually reach a deep tumor [2].

Transport of energetic charged particles in matter can be described either by deterministic codes, based on Boltzmanntype transport equations, or by Monte Carlo (MC) codes that sample the interaction process on a event-by-event basis, and both approaches rely on nuclear interaction cross-sections. The main applications of these transport codes for light ions $(Z \leq 10)$ at medium energy (100-400 MeV/nucleon) are particle therapy in oncology [3] and radiation protection in space missions [4]. The cross-sections required for transport involve total yields and multiplicities and inclusive secondary energy spectra for onedimensional transport or inclusive double-differential cross-sections in angle and energy for three-dimensional transport. For MC simulations, exclusive cross-sections may be needed for computer algorithms.

Treatment plans are generally based on deterministic codes such as the TRiP developed at GSI [5,6] or HIBRAC [7], but the great accuracy $(\leq 3 \%)$ required for medical treatment planning and sparing of normal tissues surrounding the tumors makes necessary several inter-comparisons of the codes with $\mathrm{MC}$ calculations [8-12]. All these calculations are based on measured nuclear fragmentation cross-sections of carbon ions in water or tissue-equivalent materials, mostly performed in the past in USA (BEVALAC and Berkeley), Japan (HIMAC in Chiba) and GSI in Germany (for a review see [13]). Most of these measurements are however limited to yields or total charge-changing fragmentation cross-sections, while the needed measurements of double-differential cross-section are scarce.

Not surprisingly, while fluence and total cross-sections are well described by current computer codes, the production of light fragments and their angular distribution is affected by large uncertainties and various codes may differ up to an order of magnitude in their predictions [14]. Similar problems are found in codes used for space radiation transport in shielding materials: despite several measurements of the fragmentation cross-sections related with space radio protection (reviewed in [15]), the angular distributions are not yet well reproduced. The same goes for the production of different He isotopes, mesons, and $\gamma$ rays.

NASA is currently completing a large database [16] of measured nuclear fragmentation cross-sections including approximately 50,000 datasets, and has concluded that several experimental data are missing, including double-differential cross-sections for C-ions at energies below $400 \mathrm{MeV} /$ nucleon, which are those needed also for improving treatment planning in therapy. It is therefore concluded that accurate measurements of double-differential cross-sections of light ions in the energy range $100-500 \mathrm{MeV} /$ nucleon are urgently needed for improving transport codes to be used in cancer therapy and space radiation protection.
When a light ion impinges on a target nucleus, a fragmentation process can take place depending on the impact parameter between the colliding nuclei. The target fragments usually carry little momentum, while, in particular at high energies, the projectile fragments travel at nearly the same velocity as the beam ions and have only a small deflection, except for the lighter fragments (particularly protons and neutrons). In Figs. 1 and 2, the energy and angular distribution predicted by FLUKA Monte Carlo [17,18] for the fragments produced by a $400 \mathrm{MeV} /$ nucleon carbon beam on graphite are shown: the number of all the particles produced in the target for a given run in a certain energy bin $\left(N_{\text {prod }}\right)$ divided by the number of initial C-ions $\left(N_{\text {primc }}\right)$, normalized by the bin width $(\mathrm{MeV} / \mathrm{n}, \mathrm{sr})$ for the energy (angular) spectra, are shown as a function of the fragment energy (angle). As it can be seen the heavy fragments are forward peaked and keep the projectile velocity, while a huge amount of neutrons and protons are spread out over a wide range of angle and energy.

The FIRST setup [19], located at the Heavy Ion Synchrotron SIS of GSI in Darmstad, fulfills several requirements, as shown in the next section: suitable particle identification capability providing a $\Delta M / M \leq 10 \%$ (where Mis the fragment mass), tracking capability to measure angles and momenta of the produced charged fragments, large angular acceptance for low energy protons, and finally angular acceptance for the forward produced neutrons.

A $10 \%$ relative error on the fragment mass is mandatory in order to have a clear separation of all the ions and isotopes under study. The requirement on the fragment mass separation directly translates into performance requirements (time and momentum resolution) for all the detectors that are used in the FIRST setup. The mass measured in the spectrometer is given by $M=k|R| f(\beta)$ where $k=0.3 Z / m_{0}$ and $f(\beta)=\sqrt{1-\beta^{2}} / \beta=1 / \beta \gamma$. The relative error on $M$ is hence related to the time and momentum resolutions by the relation

$\frac{(\Delta M)^{2}}{M^{2}}=\frac{(\Delta p)^{2}}{p^{2}}+\gamma^{2} \frac{(\Delta t)^{2}}{t^{2}}$

where $(\Delta p)^{2} / p^{2}=(\Delta R)^{2} / R^{2}$ has been used.

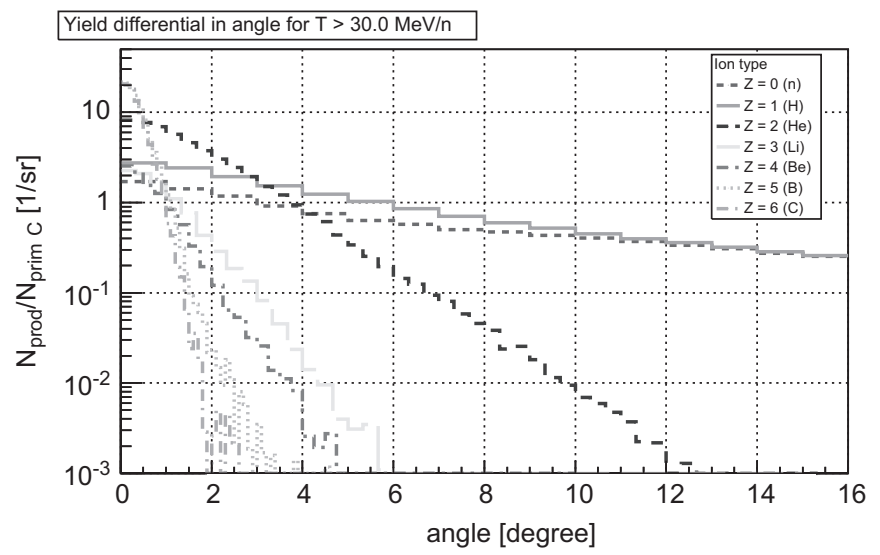

Fig. 1. Angular distribution of the fragments produced by a $400 \mathrm{MeV} /$ nucleon carbon beam on $8 \mathrm{~mm}$ carbon target. $N_{\text {prod }} / N_{\text {primc }}$ is the yield of fragments per primary carbon ion and steradian with a kinetic energy larger than $30 \mathrm{MeV} /$ nucleon (FLUKA Monte Carlo). 


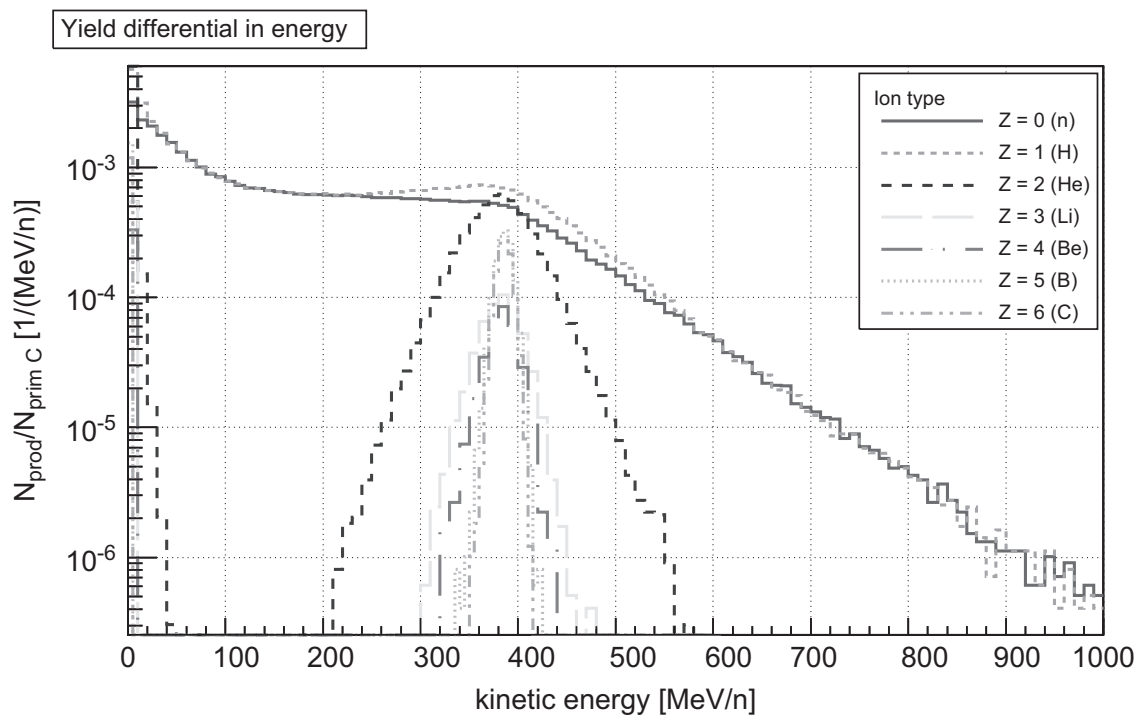

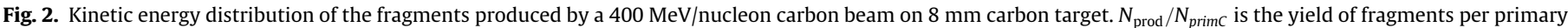
carbon ion (FLUKA Monte Carlo).

Table 1

Overview of subdetectors of the FIRST experiment.

\begin{tabular}{|c|c|c|c|c|}
\hline Name & Type & Function & Angular coverage $\left(^{\circ}\right)$ & Triggering capability \\
\hline \multicolumn{5}{|c|}{ Interaction Region (before bending by ALADiN spectrometer) } \\
\hline Start Counter & Scintillator & Start of TOF & & Yes \\
\hline Beam Monitor & Multi-wire drift chamber & Beam direction and impact point on target & & No \\
\hline Vertex Detector & Silicon pixel detector & Fragment emission angle from target & $\lesssim 40$ & No \\
\hline KENTROS & Scintillator & TOF, $\Delta E$ and coarse spatial resolution & $\approx 5-90$ & Yes \\
\hline \multicolumn{5}{|c|}{ Large Detector Region (after bending by ALADiN spectrometer) } \\
\hline TP-MUSIC IV & Time projection chamber & $\Delta E$, fragment tracking after bending & $\lesssim 5$ & No \\
\hline TOFWALL & Scintillator & Stop of TOF, $\Delta E$ and coarse spatial resolution & $\lesssim 5$ & Yes \\
\hline Veto Counter & Scintillator & Trigger veto, TOF, $\Delta E$ & $\lesssim 1$ & Yes \\
\hline LAND & Scintillator & Neutron detector, TOF, $\Delta E$ and coarse spatial resolution & $\lesssim 10$ & Yes \\
\hline
\end{tabular}

Once the fragments are properly identified, the main goal for the FIRST experiment is to provide a tabular reference for the double differential cross-sections in the energy, angle phase space. The Treatment Planning System (TPS) application of such measurements is driving the constraints on the precision that has to be reached. Aiming for a $3 \%$ uncertainty in each bin of the energy and angle $20 \times 20$ phase space (conservative assumption that actually holds only for voxels where the largest dose is released), with a $5 \%$ probability of on target interaction and a total trigger and reconstruction efficiency of $10 \%$, a total of 10-20 millions of ions on target has foreseen.

\section{The experimental setup}

The detector consists of several subdetectors divided in two main blocks: the Interaction Region and the Large Detector Region (see Table 1). The two regions are very different in dimensions of the corresponding detectors: the impinging beam and produced fragments are studied in the Interaction Region within some tens of centimeters from the target, while the devices that detect the fragments, after magnetic bending, in the Large Detector Region have typical dimension of meters

A schematic view of the FIRST experiment setup is shown in Fig. 3. Following the beam path, the Interaction Region is made of a Start Counter (SC) scintillator that provides the start to the time of flight (TOF) measurement, a drift chamber Beam Monitor (BM)

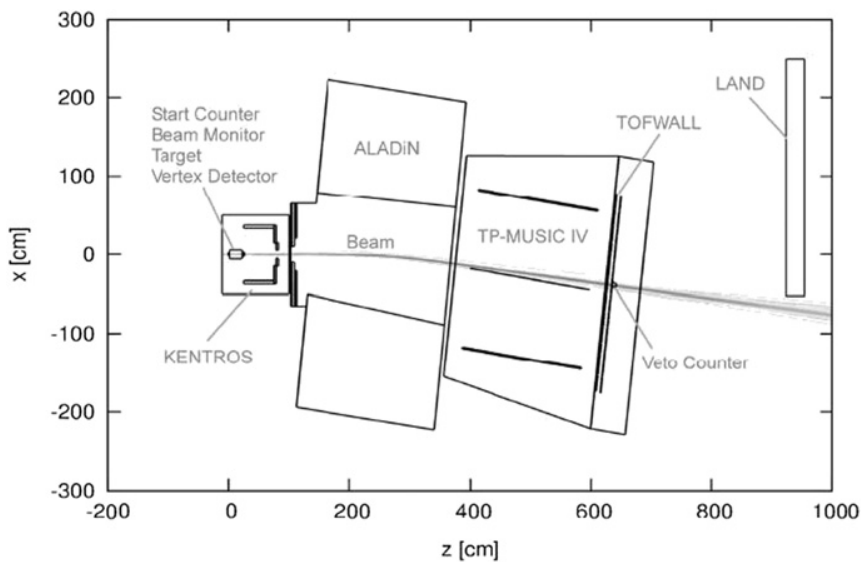

Fig. 3. Top view of the implementation of the FIRST setup. The line shows the path of the non-interacting beam particles.

that measures the beam trajectory and impact point on the target, a robotized target system, a pixel silicon Vertex Detector (VD), to track the charged fragments emerging from the thin target and finally a thick scintillator Proton Tagger (KENTROS) that detects the light fragments at large angles. The Interaction Region (IR) is in air: this choice greatly helps the design and the running of the IR detectors. On the other hand the small IR volume without 
vacuum increases the out of target interaction probability only by about $5 \%$.

With the noticeable exception of the large angle protons and a little fraction of ${ }^{4} \mathrm{He}$, most of the projectile fragments are produced in the forward direction with the same $\beta$ of the beam (see Fig. 1). These $Z \geq 2$ fragments are within the magnetic acceptance of the ALADiN dipole magnet and after magnetic bending they enter in the Large Detector Region being detected by the large volume time projection chamber (TP-MUSIC IV) that measures track directions and energy releases. A large area system of scintillators (TOFWALL) provides the measurement of the impinging point and the arrival time of the particles. The Veto Counter, a scintillator sandwich positioned after the TOFWALL in correspondence of the non-interacting beam path, is used to analyze the beam. Finally, the Large Area Neutron Detector (LAND), made of a stack of scintillator counters, gives information about the neutrons emitted within an angle of $\simeq 10^{\circ}$ with respect to the beam.

The tracking before and after the magnetic bending, coupled with the knowledge of beam direction and impact point on the target, can provide information on the $p / Z$ ratio of the produced fragments.

Care must be taken to match the information of the Interaction Region with that collected in the Large Detector Region, in particular joining the charged ion tracks, detected both by VD and by TP-MUSIC IV, with the clusters in the TOFWALL. A careful alignment can be achieved between these tracking devices using the copious events with non-interacting carbon ions.

The main features of the beam provided by the SIS accelerator are a rate of incoming particles in the range of the $\mathrm{kHz}$ and a Gaussian shape in the transverse plane of $\simeq 2.1 \mathrm{~mm}$ size $(\sigma)$. The time structure of the provided spill has a flat shape of $\simeq 10 \mathrm{~s}$ out of $20 \mathrm{~s}$ duration in total.

\section{The Interaction Region}

In Fig. 4 we show a technical drawing of the Interaction Region. All the detectors of this region have been tested at the $80 \mathrm{MeV} /$ nucleon ${ }^{12} \mathrm{C}$ beam of the Superconducting Cyclotron at the Laboratori Nazionali del Sud (LNS) of the INFN or at the Beam Test Facility $510 \mathrm{MeV}$ electron beam of the Frascati National Laboratory of INFN.

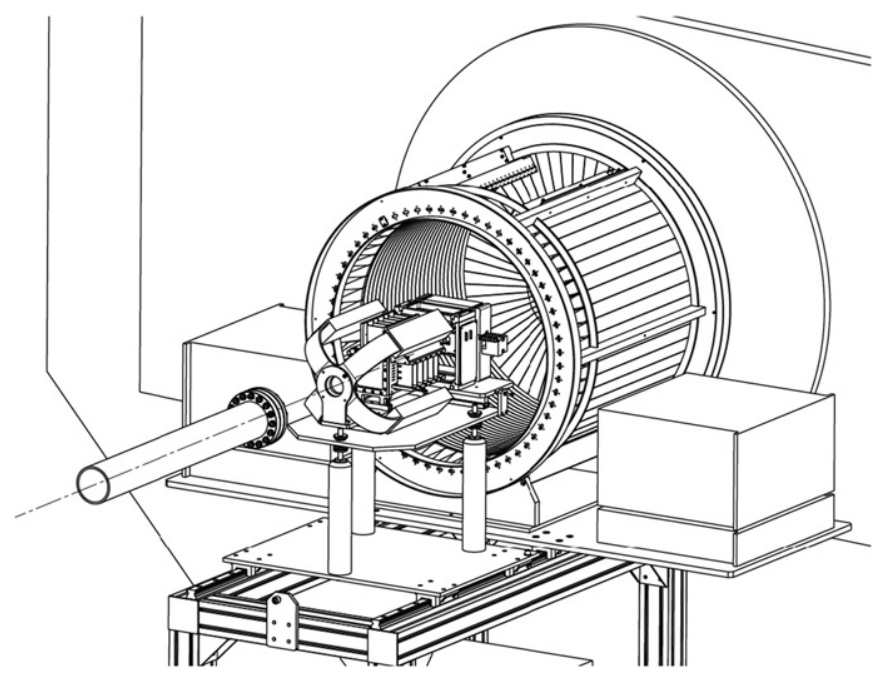

Fig. 4. Technical drawing of the Interaction Region, embedding the Start Counter, the Beam Monitor, the Vertex Detector and the Proton Tagger. The Interaction Region is located at the entrance of the ALADiN Magnet.

\subsection{The Start Counter}

The Start Counter is a thin scintillator located on the beam path $20 \mathrm{~cm}$ before the target. This device has the duty to measure the arrival time of a beam projectile and to provide a signal to the experiment trigger. In order to fulfill the requested precision on fragment TOF measurement, and hence achieve a $10 \%$ relative error on the fragment masses (see Eq. (1)), a time resolution better than $250 \mathrm{ps}(\sigma)$ must be achieved. Such resolution will also allow the measurement of the kinetic energy of the fragments detected in the proton tagger where a few ns time of flight is expected (flight path $<80 \mathrm{~cm}$ ). The scintillator must be as thin as possible to avoid beam fragmentation before the target.

In Fig. 5 the SC made of a circular thin foil of EJ228, $390 \mathrm{~nm}$ scintillator, with a diameter of $52 \mathrm{~mm}$ and a thickness of $150 \mu \mathrm{m}$, is shown. The light produced by the scintillator is collected radially by a crown of 160 optical fibers, $1 \mathrm{~mm}$ diameter each. The fibers are then grouped in four bundles that are read by fast PMT Hamamatsu H10721-210 with 40\% quantum efficiency and a time resolution of $250 \mathrm{ps} / \sqrt{N_{\text {ph.el. }}}$. The SC has been tested at LNS, on $80 \mathrm{MeV} / \mathrm{u}{ }^{12} \mathrm{C}$ and protons beams. The choice of the particles and energies was driven by the need to test the detector performances with particles with lesser (protons) and higher (carbons) energy release.

An efficiency in the range $>99 \%$ (95\%) has been obtained on carbon (proton) beams requiring a majority of three PMT signals out of four. A time resolution of $130 \pm 1$ (stat) and $310 \pm 1$ (stat) ps was obtained on ${ }^{12} \mathrm{C}$ and proton beams applying a threshold of 120 and $30 \mathrm{mV}$, respectively. These test beam results are ensuring that the performances required by the FIRST experiment can be met on the GSI carbon beam $\left({ }^{12} \mathrm{C}\right.$ of $\left.400 \mathrm{MeV} / \mathrm{u}\right)$.

\subsection{The Beam Monitor}

The Beam Monitor is a drift chamber providing two orthogonal profiles of the beam, each view detected by six planes of three cells, for a total of 36 sense wires. The cell shape is rectangular $\left(10 \times 16 \mathrm{~mm}^{2}\right)$ with the long side orthogonal to the beam, in order to minimize the possibility of an interaction of the beam with the wires. The active volume of the chamber is $2.4 \times 2.4 \times 14 \mathrm{~cm}^{3}$. The chamber is operated on carbon beam at a working point of $1.8 \mathrm{kV}$ in Argon $/ \mathrm{CO}_{2}, 80 / 20$ gas mixture. A technical drawing of the chamber is shown in Fig. 6.

The main task of the BM is the tracking of the arriving carbon, with a precision of the order of $\simeq 100 \mu \mathrm{m}$ on the impact point on the target. This resolution is needed to discriminate between double carbon tracks that can be registered in a single event by
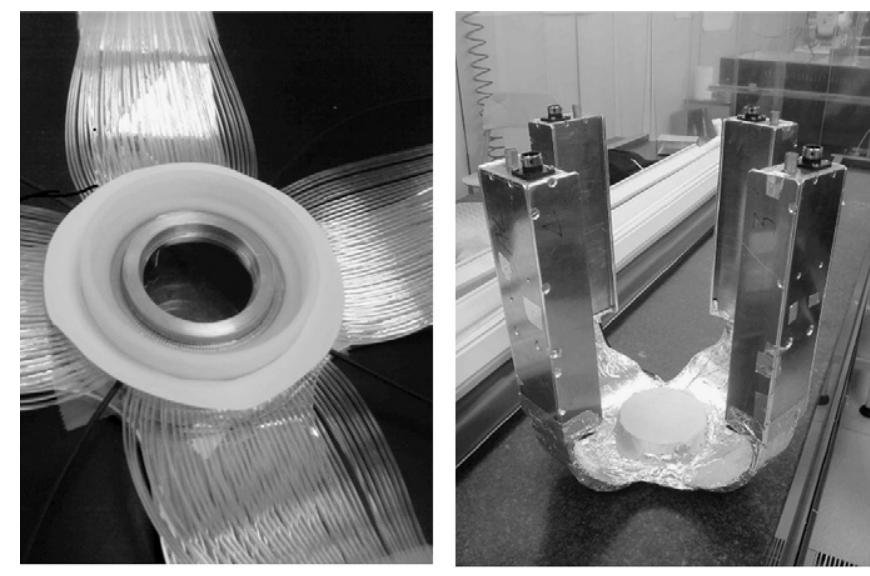

Fig. 5. The thin scintillator foil of the Start Counter read out by scintillating fibers. 


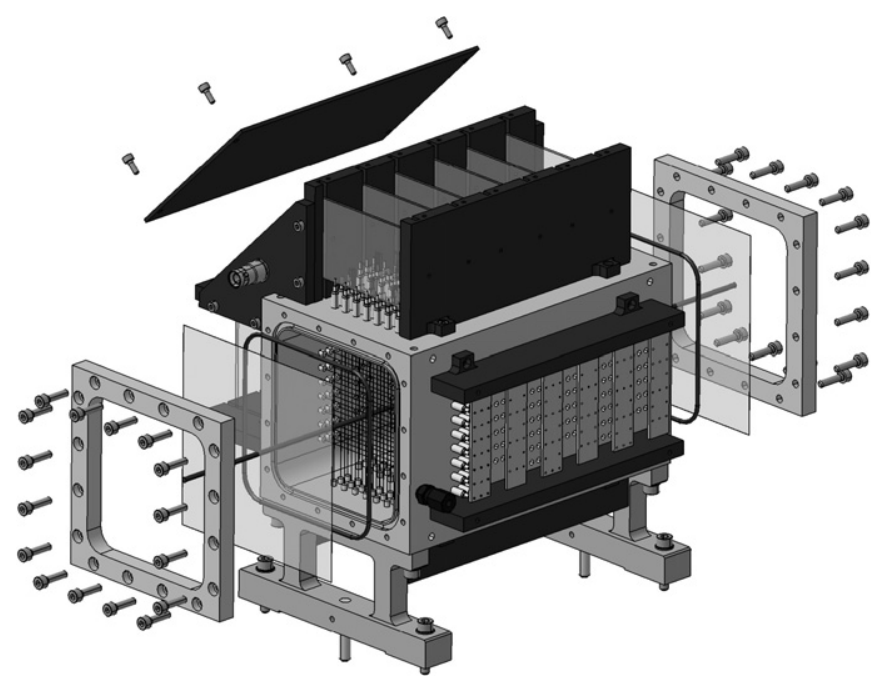

Fig. 6. Technical drawing of the Beam Monitor Drift chamber.

the slower vertex detector with $10 \%$ probability at $1 \mathrm{kHz}$ beam rate. The beam track detected by the BM in these events must point to the correct carbon track in the VD, where clusters of $100 \mu \mathrm{m}$ size are expected (see Section 3.3). This single hit space resolution of the chamber is also needed to provide a good angular resolution on the scattering angle between the carbon projectile and the fragments produced.

The BM has been tested at LNS, on $80 \mathrm{MeV} / \mathrm{u}{ }^{12} \mathrm{C}$ and protons beams and with $510 \mathrm{MeV}$ electrons at INFN LNF Beam Test Facility. The choice of the particles and energies was driven by the need to test the detector performances with particles with lesser (protons) and higher (carbons) ionization and with minimum ionizing particles (electrons). Several high voltages and gas mixtures were tested: the single hit space resolution and plane efficiency have been measured in a wide range of working conditions and we found that for all particles and beam conditions an efficiency $>99 \%$ and a spatial resolution of the order of $100 \mu \mathrm{m}$ was obtained, ensuring that FIRST requirements could be met on carbon beams in the $200-400 \mathrm{MeV} / \mathrm{u}$ energy range. The most suitable working point, based on the test beams result, appears to be the one with high voltage in the $1.7-1.8 \mathrm{kV}$ range and $80 / 20 \% \mathrm{Ar} / \mathrm{CO}_{2}$ gas mixture where an efficiency of $99 \%$ and spatial resolution of $80 \mu \mathrm{m}$ were measured.

\subsection{The Vertex Detector}

The Vertex Detector must fulfill several requirements. Even considering the non-negligible transverse size of the beam spot ( $\simeq 5 \mathrm{~mm}$ ), a wide angular coverage is needed to track also large angle projectiles that do not enter the ALADiN region. The angular resolution on tracks direction needs to be measured with an accuracy of $\approx 0.3^{\circ}$, driven by the TPS requirements of a clear separation of the $\sim 5 \mathrm{~mm}$ cubic voxels at a distance of $15 \mathrm{~cm}$. A good two track separation, with a precision at few \% level, is needed to minimize reconstruction systematic errors and an overall thickness of few \% with respect to target thickness $(\simeq 0.5 \mathrm{~cm})$ is required in order to reduce the out of target interactions. Finally a wide dynamic range is preferable in order to be able to detect both minimum ionizing particles and carbon ions of the beam.

We adopted the MIMOSA26 [20,21] pixel sensor to equip the vertex detector. MIMOSA26 has a sensitive area of $10.6 \times 21.2 \mathrm{~mm}^{2}$, subdivided in 576 rows and 1152 columns of pixels with $18.4 \mu \mathrm{m}$ pitch with a frame readout time, in rolling shutter mode, of $115.2 \mu$ s. The sensor, that provides only digital information on fired pixels, is equipped with zero suppression logic to reduce the DAQ bandwidth. Information from the BM detector will be used to identify the on target interaction, and hence the fragmentation vertex, for each event, allowing to properly associate the different tracks and clusters, in pile up events, to their origin vertex.

The vertex detector is made of four stations, each one housing two MIMOSA26s sensors glued on the two sides of a printed circuit board in correspondence to a square hole below the $2 \times 2 \mathrm{~cm}^{2}$ sensor area. The use of $1 \mathrm{~mm}$ thick printed circuit board and low profile components allows a distance between two consecutive boards of $2 \mathrm{~mm}$, with a total longitudinal dimension of the four vertex stations of $12 \mathrm{~mm}$, and an angular coverage of $\pm 40^{\circ}$. The use of a $50 \mu \mathrm{m}$ thin sensor (overall sensors thickness of $200 \mu \mathrm{m}$ ) allows to reduce the secondary fragmentation in the vertex detector, that would result in a systematic over estimation of the fragmentation cross-sections that has to be corrected by using the experiment simulation, below the desired level of a few \%. The mechanical arrangement implemented is shown in Fig. 7.

Since the MIMOSA26 sensor was not yet used to detect light ions before, we tested the sensor at LNS, exposing the MIMOSA26 to carbon beams of five different energies: 3.6, 17.2, 24.9, 31.0, $52.8 \mathrm{MeV} /$ nucleon. The results [22] reported in Fig. 8 show a clear dependence with respect the release energy, and a pixels/cluster value ranging from 50 to 100 . The measured relation justifies the scaling of the cluster size for the lighter ions. We remark that the very large clusters of $\approx 100$ pixels measured for a ${ }^{12} \mathrm{C}$ at $18 \mathrm{MeV} /$ nucleon energy are an upper bound with respect to the cluster size expected in the actual data taking. Moreover the measured cluster size indicates that we can achieve a double track separation of $99 \%$ for the expected fragments, with a maximum diameter of $\approx 6-7$ pixels $(\approx 100 \mu \mathrm{m})$, since simulation shows that clusters from a track pair will be closer than $100 \mu \mathrm{m}$ only in $0.3 \%$ of cases.

\subsection{The Proton Tagger}

The KENTROS (Kinetic ENergy and Time Resolution Optimized on Scintillator) detector, placed between the vertex detector and the ALADiN magnet, is aimed at kinetic energy and TOF measurement of light charged particles produced at polar angles larger
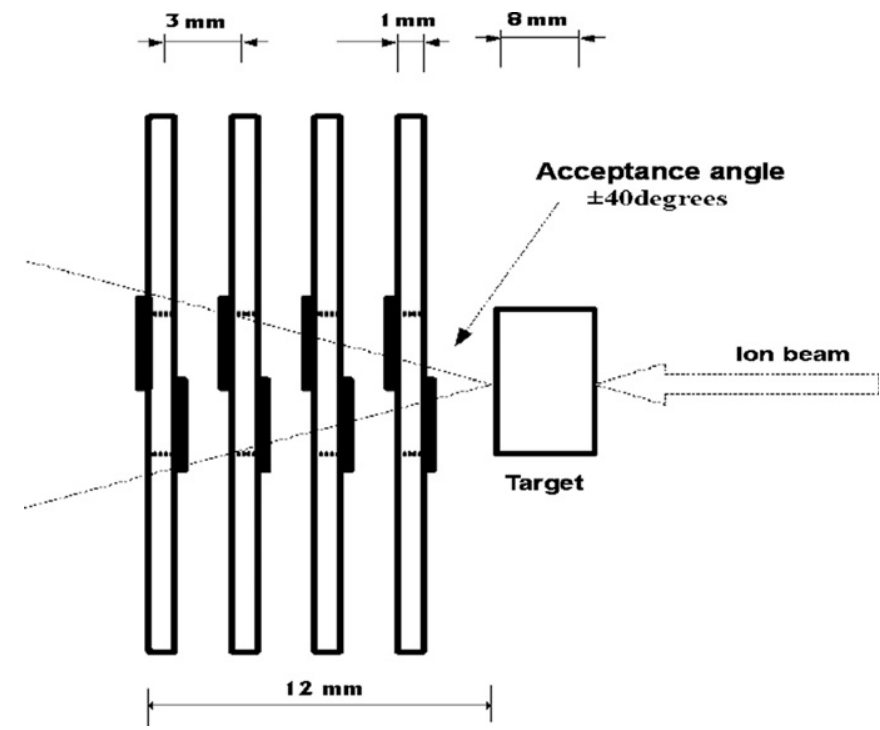

Fig. 7. Relative positions of beam, target, four vertex stations and sensor housing board dimensions. 


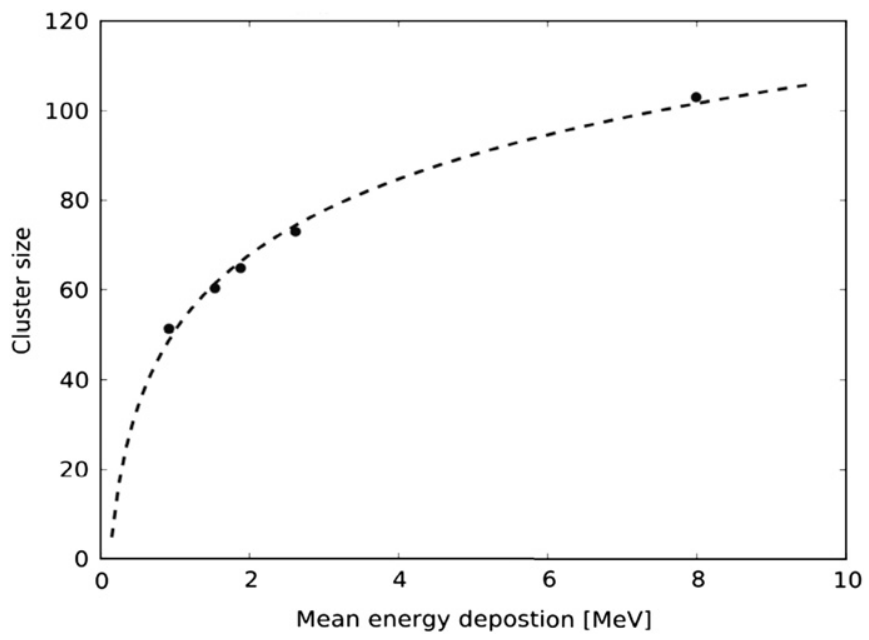

Fig. 8. Cluster size versus carbon released energy in MIMOSA26 sensor.

than about $5^{\circ}$. The response of the detector is optimized for the detection of the low energy protons. The kinetic energy of the charged particles reaching the detector can be estimated by the energy deposition and by TOF. In order to achieve a $<15 \%$ relative error on protons with kinetic energies up to $100 \mathrm{MeV}$, that are the most interesting from a TPS perspective since they release their full energy inside the patient body, the detector (geometry and electronics readout) has been designed in order to provide a time resolution of $250 \mathrm{ps}$.

The active part of the KENTROS is made of organic scintillator modules and scintillating fibers. The modules are made of EJ-200 fast scintillator, which has a decay time of $2.1 \mathrm{~ns}, 10,000$ photons/ $\mathrm{MeV}$ light yield, $425 \mathrm{~nm}$ wavelength of maximum emission and $4 \mathrm{~m}$ attenuation length. The scintillating fibers are $1 \mathrm{~mm}$ diameter BCF-10 fibers by Bicron. The scintillation light is driven from the scintillator modules to silicon photomultipliers (SIPM) using plexiglass light guides. The SIPM used are made by AdvanSiD and have $4 \times 4 \mathrm{~mm}^{2}$ active area. The SIPM output is processed by a custom electronics that amplifies, reshapes, splits and discriminates the signals in order to properly feed them in TDCs, ADCs and to provide a discriminated OR-ed signal for triggering purpose. Custom electronics have also been developed to handle the SIPM power supply and the discrimination thresholds of all the channels. The detector, shown in Fig. 9, has a cylindrical shape, and consists of three main parts.

- A barrel (Fig. 9d), which detects particles with polar angle between $36^{\circ}$ and $90^{\circ}$. The external barrel diameter is $74 \mathrm{~cm}$, and it is made of 50 scintillator modules, $3.8 \mathrm{~cm}$ thick, oriented in a direction parallel to the beam. Internally with respect to the barrel there are two layers of $1 \mathrm{~mm}$ diameter scintillating fibers that are bent to form circles in the polar region from $40^{\circ}$ to $90^{\circ}$, providing a resolution on the azimuthal angle $\sigma_{\phi} \simeq 2^{\circ}$.

- A big endcap (Fig. 9c) covering polar angles between $15^{\circ}$ and $36^{\circ}$. This device has the shape of a disk with a hole, with internal and external diameters of 28 and $74 \mathrm{~cm}$, respectively. It is composed of 60 trapezoidal scintillator modules, having $3.5 \mathrm{~cm}$ thickness along the beam direction.

- A small endcap (Fig. 9b), for particles with polar angle between $5^{\circ}$ and $15^{\circ}$. This device has $10 \mathrm{~cm}$ internal and $30 \mathrm{~cm}$ external diameter. It is composed by 24 trapezoidal scintillator modules, having $3.5 \mathrm{~cm}$ thickness along the beam direction.

The TOF resolution is affected by the scintillator decay time and light yield, by the spread of the path length of the photons in the scintillator modules and by the geometric efficiency of a

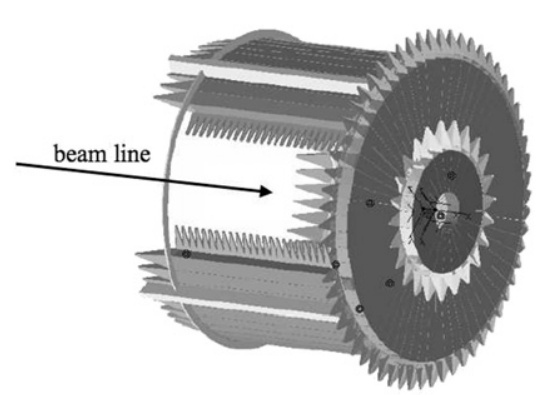

C

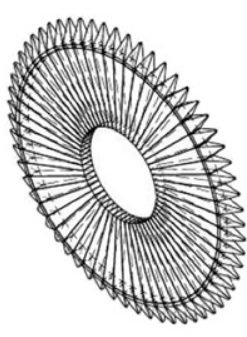

b
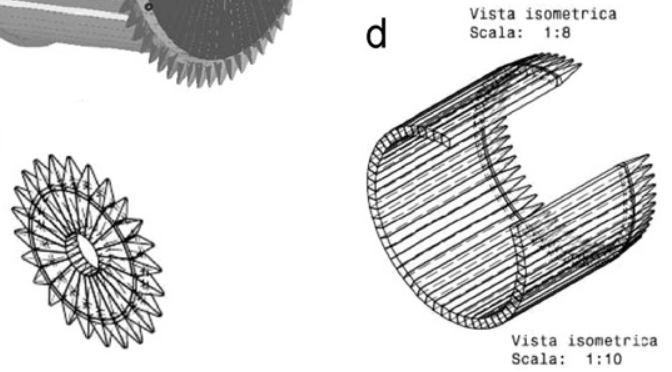

Fig. 9. Technical drawings of the KENTROS Proton Tagger. a) general assembly, (b) small endcap, (c) big endcap and (d) barrel.

scintillation light collection. It also heavily depends on the sampling of the released energy. A detailed MC simulation of the detector has been used to optimize the design and electronics in order to achieve an overall resolution on the TOF measurement of $\sigma_{\mathrm{TOF}}=270 \mathrm{ps}$ for a typical $200 \mathrm{MeV}$ kinetic energy proton (including the contribution of the time resolution of the Start Counter).

Prototypes of big and small endcap modules have been tested at the Beam Test Facility of the Frascati National Laboratory of INFN, with $510 \mathrm{MeV}$ electron beam. The measured time resolution is $350 \pm 25$ ps. This result is in fair agreement with the reported simulation result, taking into account that typical protons with $200 \mathrm{MeV}$ kinetic energy has an energy release three times higher than the BTF electrons and that the main contribution to the TOF resolution comes from the sampling of the released energy, thus scaling as $1 / \sqrt{E_{\text {rel }}}$.

KENTROS is able to determine the kinetic energy of low energy protons not only with the TOF measurement, but also using the strong correlation between the deposited energy and the $\beta$ of the particle. In the energy range of the protons produced in the FIRST experiment, the energy resolution is expected to range from a few percents for protons having $100 \mathrm{MeV}$ kinetic energy to slightly more than $10 \%$ for $400 \mathrm{MeV}$ kinetic energy protons.

\section{The Large Detector Region}

The fragments produced in the forward direction in the target enter in the large detector region crossing the ALADiN [23] dipole magnet. The ALADiN field bends the charged fragments trajectory providing information about their charges and momenta. The ALADiN magnet and the other detectors of the Large Detector Region have been inherited from previous GSI experiment and have been described in detail elsewhere $[23,29]$. Here we recall their main features as far as they are needed to describe the performance of the FIRST setup.

\subsection{The TP-MUSIC IV time projection chamber}

The TP-MUSIC IV [24] (Time Projection Multiple Sampling Ionization Chamber) detector is a tracking chamber, able to measure the charge and the momentum of nuclei from He up to 
Au with high efficiency and high resolution [25]. A cathode plane in the middle separates the active volume of the detector into two distinct drift regions with ionization chamber sections and proportional counters on each side [26]. The position of the ionizing particles in the non-bending plane is determined from the position along the proportional counters whereas the position in the bending plane is determined by measuring the drift time of the ionization electrons from the track path to the proportional counters. In order to couple with the large dynamic range of the detector and to disentangle the different particles in multiple-hit events, 14-bit FADC's digitize the signals coming directly from the preamplifiers. The device is operated in a P10 (10\% Methane and 90\% Argon) gas mixture.

\subsection{The TOFWALL}

The TOFWALL [27] detector is mainly used to measure the TOF of all fragments. It is located at $6.5 \mathrm{~m}$ from the target, behind the TP-MUSIC IV, and covers the angular range $0^{\circ}<\theta<6.5^{\circ}$. The whole detector is inside a chamber filled with nitrogen and separated from the chamber containing the TP-MUSIC IV by a mylar window. The TOFWALL is composed by two detector layers (front and back), perpendicular to the beam, each made of 12 modules. Each module is built up of eight plastic scintillators (BC408), $1.10 \mathrm{~m}$ long, $2.5 \mathrm{~cm}$ wide and $1 \mathrm{~cm}$ thick, and is covered with an aluminum foil. A brass foil $0.5 \mathrm{~mm}$ thick between the two layers shields the last layer from the $\delta$ electrons generated in the first layer, in order to improve the charge identification. The two layers are shifted from each other of $1.25 \mathrm{~cm}$, corresponding to half a scintillator slat, in order to maximize the probability that incoming fragments hit at least one slat. Each slat is connected to a photomultiplier at each side. The signals are digitized using Fastbus QDCs to extract the energy information and TDCs for the time measurement. In past experiments [27], a time resolution of about 170 and $80 \mathrm{ps}$ for fragments of $Z=2$ and $Z=12$, respectively, was achieved and elements with $Z \leq 15$ were resolved individually.

\subsection{The LAND detector}

The Large Area Neutron Detector (LAND) [28,29] is designed for neutron detection. The detector has an active area of $2 \times 2 \mathrm{~m}^{2}$ and its depth is $1 \mathrm{~m}$. It is made of a multilayer structure of passive converter and active scintillator materials. Since neutron produces recoil protons via elastic scattering, using materials rich in protons, such as plastic scintillators, the neutron can be detected by the light produced by the scattered proton.

The full detector is divided into 200 paddles of $200 \times 10 \mathrm{~cm}^{2}$ area and $10 \mathrm{~cm}$ depth. Each paddle contains 11 sheets of iron (the two outer ones are $2.5 \mathrm{~mm}$ thick, the others are $5 \mathrm{~mm}$ thick) and 10 sheets of $5 \mathrm{~mm}$ thick scintillator, mounted in an iron sheet box which has a wall thickness of $1 \mathrm{~mm}$. Twenty paddles are arranged in one layer; subsequently layers are mounted with the paddles perpendicular to each other, thus giving position information in both vertical and horizontal directions. Light produced in a paddle is collected by means of stripe light guides on both ends of the scintillator sheets and is directed to the photomultipliers. The difference in arrival time of the two signals is used to localize the position where scintillation light was produced by secondary, charged particles; the mean time provides time-of-flight information. The time resolution of the modules is $\Delta t=210-250 \mathrm{ps}(\sigma)$ and the position resolution $\Delta x=7-10 \mathrm{~cm}$. For neutrons emitted with kinetic energies in the $0.5-4 \mathrm{MeV}$ range, an energy resolution of $0.4-1 \mathrm{MeV}$ was measured.

\subsection{The Veto Counter}

The Veto Counter (VC) detector is placed after the TOFWALL on the magnetic path of the carbon projectiles that do not interact in the setup. The counter is made of two scintillator slabs (BC-404 by Saint Gobain), with parallelepiped shape and with a volume of $6 \times 6 \times 3 \mathrm{~cm}^{3}$ and $4 \times 4 \times 6 \mathrm{~cm}^{3}$, respectively. The two slabs are put one on top of the other, with the equal sides orthogonal to the carbon track and centered with respect to the carbon path. The larger slab is read by two PMT by Hamamatsu on both sides, while the little one is read from the rear direction, along the beam. The signals are readout by the same ADCs and TDCs used for KENTROS. The VC monitors the amount of fragments that are produced in the same angular range of the non-interacting beam. The Veto Counter signal is also used to label non-interesting events where the carbon projectile has not interacted and arrives unperturbed on the Veto Counter.

\section{DAQ and trigger}

The readout is handled by the Multi Branch System (MBS), a general DAQ framework developed at GSI [30]. In the MBS several intelligent bus controllers (CES RIO), running under the real-time operating system LynxOS, perform the readout of the digitization modules of the individual crates, when triggered by the dedicated trigger modules. All the trigger modules, one in each readout crate, are connected via a trigger bus to distribute the trigger and dead-time signals and to ensure event synchronisation. Data collected by single controllers are broadcast via Ethernet to an event-builder where they are merged and saved in the standard GSI format. A set of client-server applications allows to control the data acquisition, to remotely configure the detector settings and to perform on-line monitoring of the data quality. MBS can handle easily the different Front End Electronics standards used by the different subdetectors: FASTBUS, CAMAC and VME.

The expected dead time due to trigger signal formation and readout is of the order of ms per event due to several factors as drift time in the TP-MUSIC IV, conversion time in the digitization modules and transfer data time from the electronics to the readout controllers and from the controllers to the event builder via TCP/IP. An efficient trigger system is then essential to select the fragmentation events and to keep the counting rate at a level where the inefficiencies due to the dead-time are minimized.

The final trigger decision requests the coincidence of the Start Counter trigger with the trigger of any of the detectors: TOFWALL, LAND, KENTROS, and the downscaled Veto Counter. In order to suppress events in which the carbon projectile does not interact with the target, coincidences between the Start Counter and the Veto Counter can be rejected. An additional unbiased trigger condition based on the Start Counter trigger alone is also foreseen with tunable downscale factor to estimate the efficiency of the other trigger conditions.

The reported trigger logic is implemented in an FPGA programmable VME module (VULOM4 [31]). This module accepts individual trigger signals and implements all the logic matrices and the downscale factors needed to control the trigger conditions, the scalers to count input and output triggers, the internal generators of regular calibration triggers and the locking mechanism to block the propagation of triggers during the dead time. Different trigger conditions for the accepted triggers are encoded on different lines of the trigger bus and propagated to the readout electronics.

Finally, in order to avoid the delay lines needed to synchronize the KENTROS analog signals to the ADC's with the delayed master trigger decision, a two-level trigger system is implemented for the 
detectors in the interaction region. The analog signals are processed in the ADC's as soon as the local trigger from the start counter is generated; if no master trigger is received within a fixed time, a fast clear signal is provided to the electronics and the event is ignored by the DAQ system.

\section{The Monte Carlo simulation}

The simulation of the FIRST experiment provides simulated detector response data of a full event. This software not only supports design and optimization of the experimental setup, but also provides training data for the reconstruction software in the preparatory stage of the experiment. Furthermore, it facilitates the evaluation of acceptances, reconstruction efficiencies and other systematics.

The simulation of the particle transport and interactions is based on the multi-purpose MC code FLUKA. In addition to an accurate description of electromagnetic processes, FLUKA was shown to provide a modelling of nuclear interactions which is judged to be satisfactory in the energy range of FIRST [12,32].

The implementation of the simulation breaks up into several subsectors:

- description of experimental setup configuration (beam phase space, geometry and materials, parameters describing detector properties and magnetic field),

- particle transport with the MC code FLUKA and retrieval (scoring) of basic physical quantities of the tracks (i.e., primary particles and created secondary particles which are propagated through the detector geometry) and hits (i.e., energy depositions of tracks in sensitive detector elements),

- modelling of the subdetector responses and digitization,

- storing of simulated track, hit and detector signal data for further processing and analysis.

The data flow of the simulation is illustrated in Fig. 10. In order to facilitate a flexible and object-oriented coding of the geometry and processing of the MC simulation data, the FORTRAN77-based FLUKA code was interfaced with $\mathrm{C}++$. This allows the smooth conversion and storage of virtually arbitrarily large simulated event samples in the ROOT tree format [33]. The reconstruction and analysis software of the FIRST experiment reads and processes the MC events from the ROOT files.

The experimental setup was implemented including all detectors and the ALADiN spectrometer, as shown in Fig. 3. The geometry and all the materials of the detectors have been modelled with a considerable detail, i.e. including the wires of the BM, vacuum windows, the air and gas mixtures crossed in the setup, to reliably evaluate the out of target fragmentation in all the materials crossed by the carbon projectiles and the produced fragments.
Raw physical quantities, such as energy deposition, particle properties and their paths (tracks), are obtained by the MC simulation in the sensitive detector regions (scoring regions), to produce the digitized response of each detector. Starting from these basic quantities, the detector signal amplitudes and signal arrival times are computed using simple signal modelling, such as quenching effects (which can be parametrized by Birks law [34]), light attenuation and signal velocity in scintillators and drift velocities in gases. More complex signal dependencies, such as detection efficiencies and resolutions, are determined from measurements and parametrized for the simulation. This guarantees a reduced computative effort and decreases the overall complexity of the simulation, while preserving the predictive power of the simulation at a reasonable level. Finally, the resulting analog quantities are digitized and cast into a format as needed to be processed by the data reconstruction software.

FLUKA predicts a interaction probability in a $5 \mathrm{~mm}$ graphite target for a $400 \mathrm{MeV} /$ nucleon ${ }^{12} \mathrm{C}$ beam equal to $4.26 \pm 0.06 \%$ (the quoted uncertainty is statistical, one sigma). The trigger efficiency has then been evaluated by the MC to be around 95\%, while the trigger efficiency on the events where the beam interacts after the target in the detector materials is $\simeq 5 \%$. These figures slightly depend on the energy thresholds chosen for the subdetector triggers.

\section{Conclusion}

The FIRST experiment has been setup at the GSI laboratory due to the renewed interest in the light ion fragmentation in the field of tumor therapy and space radioprotection. The main features of the detector are the tracking of the produced fragments, the particle ID capability and the large angular acceptance. This last feature allows the detection of the significant component of low energy protons known to be emitted in the fragmentation process in the $0^{\circ} \leq \theta \leq 90^{\circ}$ angular region.

The experiment started its scientific program in summer 2011 with a $400 \mathrm{MeV} /$ nucleon carbon beam on an $8 \mathrm{~mm}$ graphite target during a 10 days data taking shift, and a data sample of $2 \times 10^{7}$ events has been collected with this configuration. Samples of $10^{6}$ events without the graphite target and of $10^{6}$ events without magnetic field has been taken for calibration purpose and in order to study the systematics due to the out of target fragmentation of the beam particles in the detector material. Finally, a sample of $2 \times 10^{6}$ events with $400 \mathrm{MeV} /$ nucleon carbon beam, on $0.5 \mathrm{~mm}$ gold target, has been also collected.

The setup has been designed to extract the detector efficiency, as much as possible, directly from the data. Furthermore, a detailed MC simulation tool has been developed with the specific aim of evaluating the acceptances and systematics. The union of the information from different subdetectors will allow a nearly complete reconstruction of the fragmentation reaction thus providing a deep insight of the related mechanism.

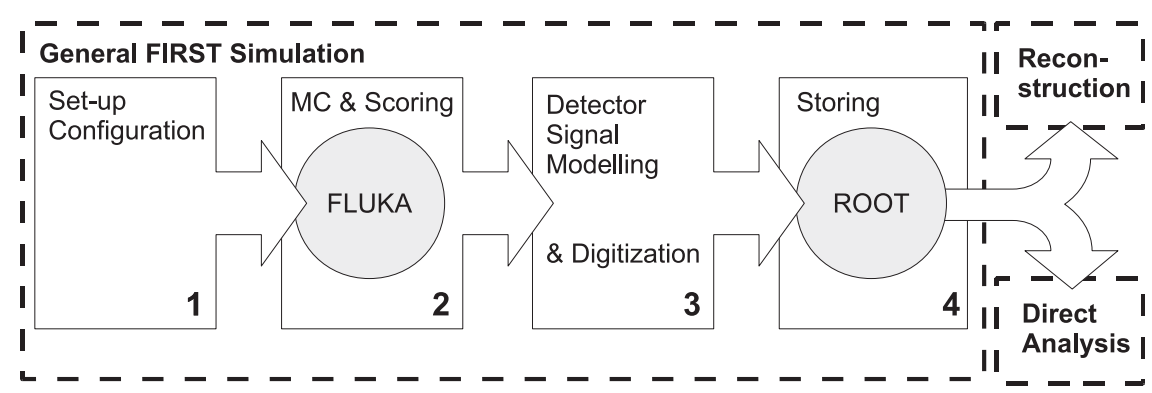

Fig. 10. Data flow of the simulation. 
After this initial data taking, the FIRST experiment could also be a facility to measure with great accuracy the fragmentation of different projectile-target combination. In particular the collaboration strongly supports possible future measurements of the $\mathrm{He}$, Li and Fe fragmentation cross-sections.

\section{Acknowledgements}

We would like to acknowledge M. Arba, L. La Delfa and M. Tuveri (INFN Sez. Cagliari), M. Anelli, S. Cerioni, G. Corradi, D. Riondino and R. Rosellini (INFN, LNF), M. Capponi and A. Iaciofano (INFN, Sez. Roma3) for the technical design and mechanical work on the Interaction Region, and Filippo Bosi (INFN Sez. Pisa) for his help and suggestions. This work has been supported by the European Community FP7 - Capacities, contract ENSAR n ${ }^{\circ} 262010$. This work was also supported by Junta de Andalucia and the Spanish Ministerio de Ciencia e Innovacion Contracts P07-FQM-02894, FIS2008-04189 and FPA2008-04972-C03. Finally some of the authors would like to thank CNRS-In2p3 for the support. The research leading to these results has received the financial support of the Belgian company Ion Beam Applications (IBA).

\section{References}

[1] O. Jäkel, et al., Medical Physics 35 (2008) 5653.

[2] S. Kox, et al., Physical Review C 35 (1987) 1678.

[3] M. Durante, J.S. Loeffler, Nature Reviews Clinical Oncology 7 (2010) 37

[4] M. Durante, F.A. Cucinotta, Nature Reviews Cancer 8 (2008) 465.

[5] M. Krämer, Nuclear Instruments and Methods in Physics Research Section B 267 (2009) 989.

[6] M. Krämer, M. Durante, The European Physical Journal D 60 (2010) 195.

[7] L. Sihver, D. Mancusi, Radiation Measurements 44 (2009) 38.
[8] F. Sommerer, et al., Physics in Medicine and Biology 51 (2006) 4385.

[9] J. Allison, et al., IEEE Transactions on Nuclear Science NS-53 (2006) 270.

[10] M.C. Morone, et al., Physics in Medicine and Biology 53 (2008) 6045.

[11] T. Sato, et al., Radiation Research 171 (2009) 107.

[12] T.T. Böhlen, et al., Physics in Medicine and Biology 55 (19) (2010) 5833.

[13] D. Schardt, et al., Reviews of Modern Physics 82 (2010) 383.

[14] J.H. Heinbockel, et al., Advances in Space Research 47 (2011) 1079.

[15] M. Durante, F.A. Cucinotta, Reviews of Modern Physics 83 (2011) 1245.

[16] J.W. Norbury, J. Miller, 47th NCRP Annual Meeting, Bethesda, MD, 2011, p. 24.

[17] G. Battistoni, et al., The FLUKA code: description and benchmarking, in: Proceedings of the Hadronic Shower Simulation Workshop 2006, AIP Conference Proceedings, vol. 896, 2007, p. 31.

[18] A. Ferrari, P.R. Sala, A. Fasso', J. Ranft, FLUKA: A Multi Particle Transport Code, Technical Report CERN-2005-10, INFN/TC05/11, SLAC-R-773, 2005.

[19] V. Patera, Nuclear physics experiment for hadrontherapy application, Nuovo Cimento C 34 (6) (2011).

[20]〈http://www.iphc.cnrs.fr/-CMOS-ILC-.html〉.

[21] G. Deptuch, et al., Nuclear Instruments and Methods in Physics Research Section A 512 (2003) 299.

[22] E. Spiriti, M. De Napoli, F. Romano, Nuclear Physics B (Proceedings Supplements) 215 (2011) 157

[23] J. Hubele, et al., Zeitschrift für Physik A 340 (1991) 263.

[24] C. Sfienti, et al., Proceedings of the XLI International Winter Meeting on Nuclear Physics, Bormio, Italy, 2003, p. 323.

[25] C. Sfienti, et al., Physical Review Letters 102 (2009) 152701.

[26] G. Bauer, et al., Nuclear Instruments and Methods in Physics Research Section A 386 (1997) 249

[27] A. Schuttauf, et al., Nuclear Physics A 607 (1996) 457.

[28] T. Blaich, et al., Nuclear Instruments and Methods in Physics Research Section A 314 (1992) 136

[29] K. Boretzky, et al., Physical Review C 68 (2003) 024317.

[30] H.G. Essel, N. Kurz, IEEE Transactions on Nuclear Science NS-47 (2) (2000) 337.

[31] 〈https://www.gsi.de/informationen/wti/ee/elekt_entwicklung/vulom_m_e. html $\rangle$.

[32] F. Sommerer, et al., Physics in Medicine and Biology 51 (17) (2006).

[33] R. Brun, F. Rademakers, Nuclear Instruments and Methods in Physics Research Section A 389 (1996) 81.

[34] J.B. Birks, The Theory and Practice of Scintillation Counting, Pergamon Press, Oxford, 1964. 